\title{
Integration of a CAS/DGS as a CAD system in the mathematics curriculum for architecture students
}

\author{
R.M. Falcón* \\ Department of Applied Mathematics I, High Technical School of Building Engineering, \\ University of Seville, Seville, Spain
}

\begin{abstract}
Students of Architecture and Building Engineering Degrees work with Computer Aided Design systems daily in order to design and model architectonic constructions. Since this kind of software is based on the creation and transformation of geometrical objects, it seems to be a useful tool in Maths classes in order to capture the attention of the students. However, users of these systems cannot display the set of formulas and equations which constitute the basis of their studio. Moreover, if they want to represent curves or surfaces starting from its corresponding equations, they have to define specific macros which require the knowledge of some computer language or they have to create a table of points in order to convert a set of nodes into polylines, polysolids or splines. More specific concepts, like, for instance, those related to differential geometry, are not implemented in this kind of software, although they are taught in our Maths classes. In a very similar virtual environment, Computer Algebra and Dynamic Geometry Systems offer the possibility of implementing several concepts which can be found in the usual mathematics curriculum for Building Engineering: curves, surfaces and calculus. Specifically, the use of sliders related to the Euler's angles and the generation of tools which project $3 \mathrm{D}$ into $2 \mathrm{D}$, facilitate the design and model of curves and rigid objects in space, by starting from their parametric equations. In this article, we show the experience carried out in an experimental and control group in the context of the Maths classes of the Building Engineering Degree of the University of Seville, where students have created their own building models by understanding and testing the usefulness of the mathematical concepts.
\end{abstract}

Keywords: dynamic geometry systems; 3D modelling; differential geometry; curves; surfaces;

\section{Introduction}

Banerjee and de Graaff state [1] that the curricula of Architecture and Building Engineering Degrees are so dominated by the study of design that those disciplines which are not based on it are perceived by students as peripheral. This is usually the case for the Mathematics discipline, which becomes a summary of a huge list of concepts and tools which are not clearly related with design. Moreover, Geometry, 
which can be the most related field, is usually taught in class in such a rigorous way that students cannot directly see its integration into their professional career.

The general proposal given by Banerjee and de Graaff is the implementation of a Problem-based Learning [2] and the collaborative interaction among disciplines in order to enrich and enhance the architectural design process. In this sense, Verner and Maor [3,4] adopt the following two approaches to teaching Mathematics in Architecture: Realistic Mathematics Education [5], in which learning mathematical concepts is based on solving applied problems related to architecture studies, and Mathematics as a Service Subject [6], where mathematical tools are used in performing architecture design assignments for professional practice. Specifically, they state [7] that geometrical forms, like tessellations, curved surfaces and solids intersections, can be used in order to study existing architectural structures and generate design alternatives.

In general, students of Architecture and Building Engineering carry out the analysis, modelling and design of architectural structures by using Computer Aided Design (CAD) systems like, for instance, AutoCAD, ${ }^{1}$ ArchiCAD $^{2}$ or Rhino. ${ }^{3}$ This kind of software is based on the creation of basic two- and three-dimensional geometrical objects, like straight lines, rectangles, arcs, ellipses, prisms, spheres, cylinders, cones or tori, which can be transformed by using symmetries, rotations, translations or homotheties. It is also possible to obtain the boolean union, intersection or difference between two of these objects. However, if users want to represent a conic which is not given as a defect tool (like, for instance, a hyperbola), then they have to define a set of points of the conic and create a polyline or a spline as an approximation. Analogously, in order to obtain a quadric, it has to be approximated by a polysolid or, if it is the case, it has to be obtained as the revolution of a polyline or spline.

It can be also observed that, in order to insert the exact mathematical equation of a curve or a surface in a CAD system, users have to define a specific macro in a computer language, like LISP, with a polyline or a polysolid as output. It causes a lack of accuracy which can be relevant in, for example, concepts like length, curvature or torsion of a curve. Indeed, these specific concepts related with differential geometry are not generally implemented in CAD systems or cannot be obtained from a drawn curve or surface.

Although it is clear that CAD systems are intrinsically based on mathematical concepts, they do not allow users to work directly with the set of formulas and equations which constitute the basis of their studio. At most, they can only introduce some generic constraints or parameters like the coordinates of the vertices of a solid, its dimensions, the scale, the display angle or, in some specific cases, several geometrical parameters like the main elements of a conic, a quadric or a plane or space transformation. In this sense, parametric CAD systems are useful, because they record the construction process and allow to change parameters at any time of the modelling process, in such a way that all the geometrical objects related to them are immediately modified according to the changes. This aspect makes it easy to model similar architectural structures in only one worksheet.

The mathematical concepts in which CAD systems are based and their generalized use in other disciplines of Architecture and Building Engineering are two reasons to keep in mind so that CAD systems can be incorporated in the mathematics curriculum. Specifically, they can be used to capture the attention of students and improve their attitudes towards Mathematics. 
In this article, we show that the use of a Dynamic Geometry System (DGS) can be a feasible solution, because of their analogy with a parametric CAD system. Specifically, DGSs, like, for instance, Cabri ${ }^{4}$ or Cirendella, ${ }^{5}$ are based on compassand-ruler constructions of basic geometrical objects, whose parameters can be changed at any time, with the immediate reconstruction of the complete worksheet. In contrast to the variety of visual effects which can be obtained using a CAD system, the default set of geometrical objects of a DGS is much more complete than that of a CAD system and, therefore, it is not necessary use polylines or splines. Moreover, there are more and more DGSs incorporating algebraic tools which allow to draw curves by knowing their corresponding equations. A DGS of this type incorporates the philosophy of a Computer Algebra System (CAS) and becomes a CAS/DGS, like, for example, GeoGebra. ${ }^{6}$

\section{Case study framework}

A learning methodology based on the use of the CAS/DGS GeoGebra has been tested in the two first-year mathematics courses of the Building Engineering Degree of the University of Seville. This degree has been recently implemented in the Academic Year 2009-2010 within the context of the Bologna Process on the European Space for Higher Education. The contents of these subjects are the following:

(i) Applied Mathematics for Building Construction I (6 ECTS): Statistics, resolution of nonlinear equations, resolution of linear equation systems, matrix calculus, linear and affine transformations, conics and quadrics.

(ii) Applied Mathematics for Building Construction II (6 ECTS): Curves, surfaces, calculus, numerical integration, optimization and differential geometry.

It can be observed that the topics of both courses are so wide and varied that a good methodology is necessary in order to cover all of them within the stipulated contract time per subject. All these topics were taught for 10 academic years within the previous Technical Architecture Degree in a common mathematics course of 135 on-site hours, where only $25 \%$ of the hours were related to computer-aided teaching. Specifically, the CAS Maple ${ }^{7}$ was used to study the set of concepts which are not so related to Geometry. In this sense, from our experience, although a CAS is a good tool to introduce concepts and do computations, our students find it difficult to work with a software based on subsequent commands and without a graphical and intuitive interface like that of a CAD system. Consequently, they need a reasonable length of time to work with a CAS and, therefore, the lack of time in the new courses was a disadvantage to take into consideration.

Consequently, we considered the possibility of complementing the use of Maple with a CAS/DGS so that our students would be motivated with a familiar environment. In this current article, we describe how they have used the CAS/DGS GeoGebra as a mathematical CAD. ${ }^{8}$ Specifically, the following tasks are detailed.

(i) Analysis of the contour of a building.

(ii) Floor plan elevation.

(iii) Implementation of tools related to differential geometry on curves.

(iv) $3 \mathrm{D}$ modelling of surfaces and architectural structures. 


\section{Analysis of architectural structures by using a CAS/DGS}

Verner and Maor [8] state that the analytical description of the contours of a building, using functions and equations depending on parameters, is a prerequisite for CAD studies. In their experimental proposal, they use pictures of buildings and graphical representations of their contours on squared article sheets. In order to improve this traditional method, the possibility of including background pictures in GeoGebra allows this analysis of contours to be directly done on the photograph of a building, with the consequent improvement in the accuracy of the approximation. Moreover, the use of sliders and algebraic tools makes possible a fast and efficient study of a parametric dependence.

In this regard, we suggest our students to take or look for a photograph of an architectural structure with a known conical contour. It is advisable to choose a conic in canonical form. Furthermore, the photo has to be taken opposite the building so that the perspective has no influence in the analysis and some of the dimensional measurement of the structure has to be known in order to study its contour. Once the photography has been included in the worksheet of GeoGebra, the following tasks are asked.

(i) Choose five points of the contour and obtain the conic which passes through these points. Check if the type of the obtained conic coincides with the known data.

(ii) Use several sliders in order to obtain the parameters of the canonical equation of the conical contour. Compare the new approximation with the previous one.

(iii) Obtain the main elements of the last conic and check their properties.

As an example, we study in class the contour of the front of the Berliner Bogen Office in Hamburg, which is an office building with the shape of a parabolic cylinder of 36 meters height (Figure 1). Once the photograph of the building is included in the worksheet in such a way that the top of the parabolic arc is at the point $(0,36)$, we start with the following tasks.

(i) We use the grid so that we can choose two pair of points of the arc which are pairwise symmetric with respect to the ordinate axis. Then, we use the command Conic through five points and we check if the obtained conic is a parabola. Since it is difficult to have a photo with a perfect perspective and symmetry, a conic which is not a parabola is usually obtained (Figure 2).

(ii) Since $(0,36)$ is the vertex of our parabola, its canonical equation will be

$$
x^{2}=-2 p \cdot(y-36),
$$

where $p / 2$ is the focal distance. Thus, we define a slider $p$ in the interval $[0,36]$ and we define in the input box the parabola $x^{2}=-2 p \cdot(y-36)$. Now, we move the slider in order to approximate the contour of our arc and we compare it with the previous approximation (Figure 3). In our case, the best approximation will be those in which $p=18$.

(iii) By using the tool Circle with centre and radius, we define the circumference of centre $(0,36)$ and radius $p / 2$. Its intersections with the ordinate axis will define the focus $F$ and the directrix line $l$ of the parabola. Finally, if we 
File Edit View Options Tools Window Help

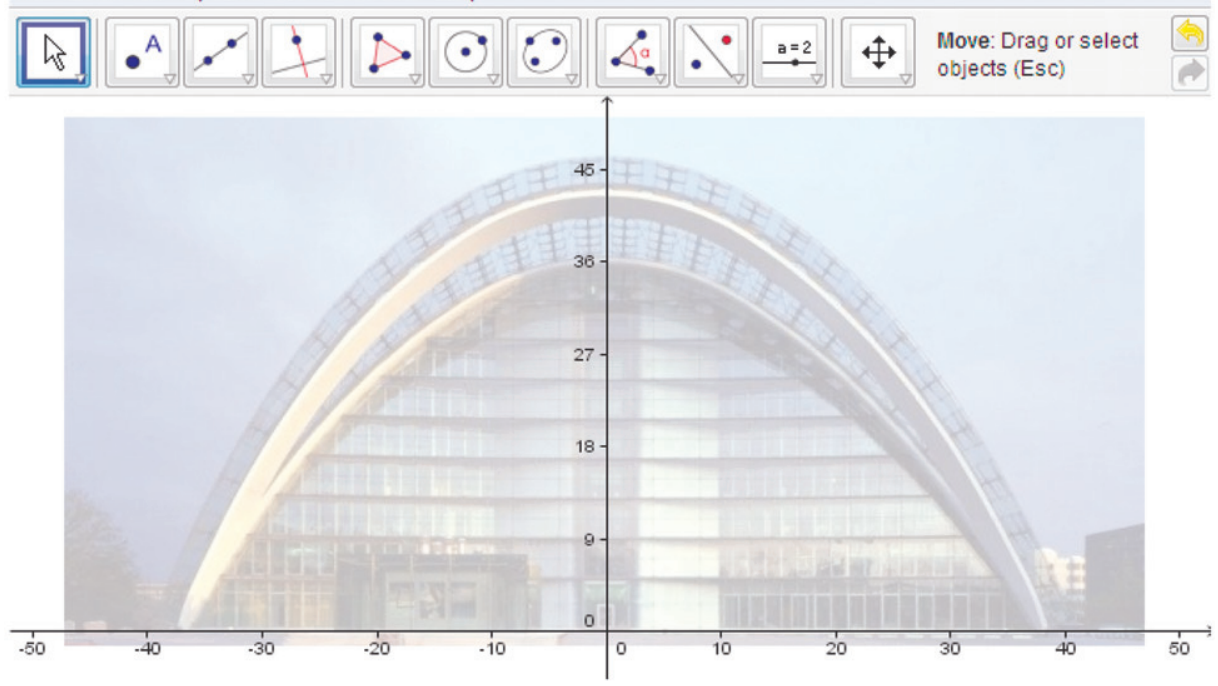

Figure 1. Berliner Bogen Office (Hamburg, Germany).

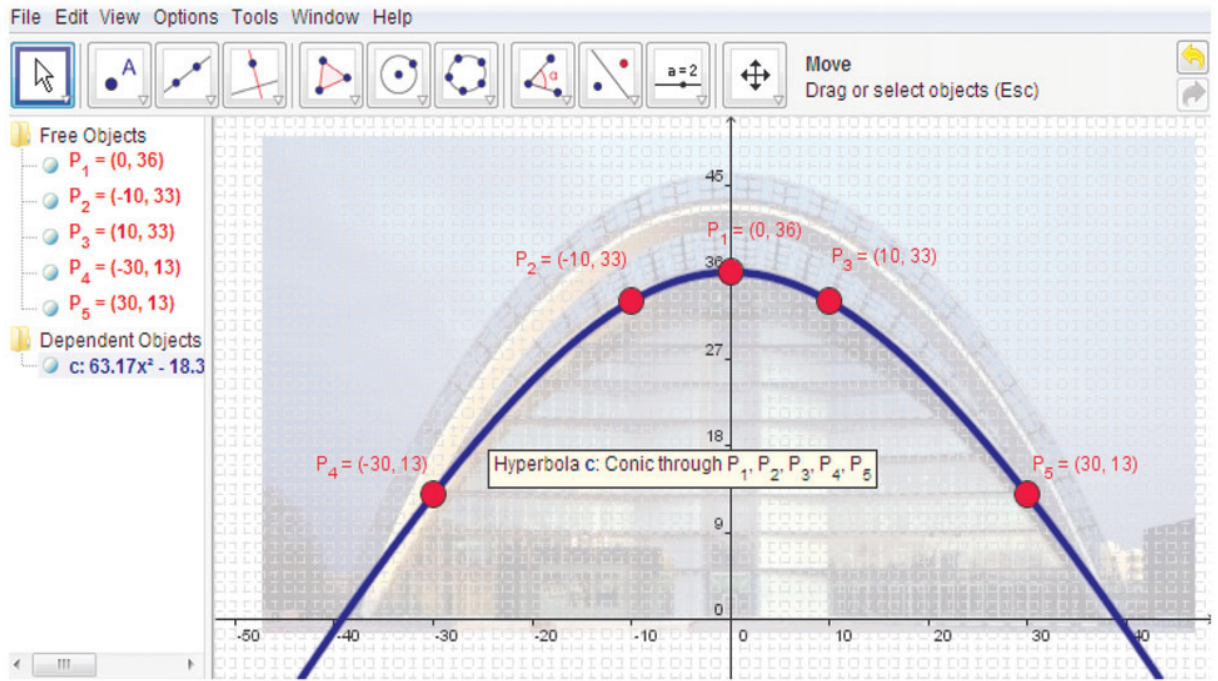

Figure 2. Approximation of the parabolic arc with a hyperbola which is obtained using the command Conic through five points.

create a point $P$ on the parabola, then we can use the tool Distance or Length and move $P$ in order to check that $d(P, F)=d(P, l)$ (Figure 4).

Let us remark that, even if the previous example is the first one which is set out in class, its resolution is done in a collaborative way between teacher and students and some unexpected but interesting questions can appear. In this regard, the following 
File Edit View Options Tools Window Help

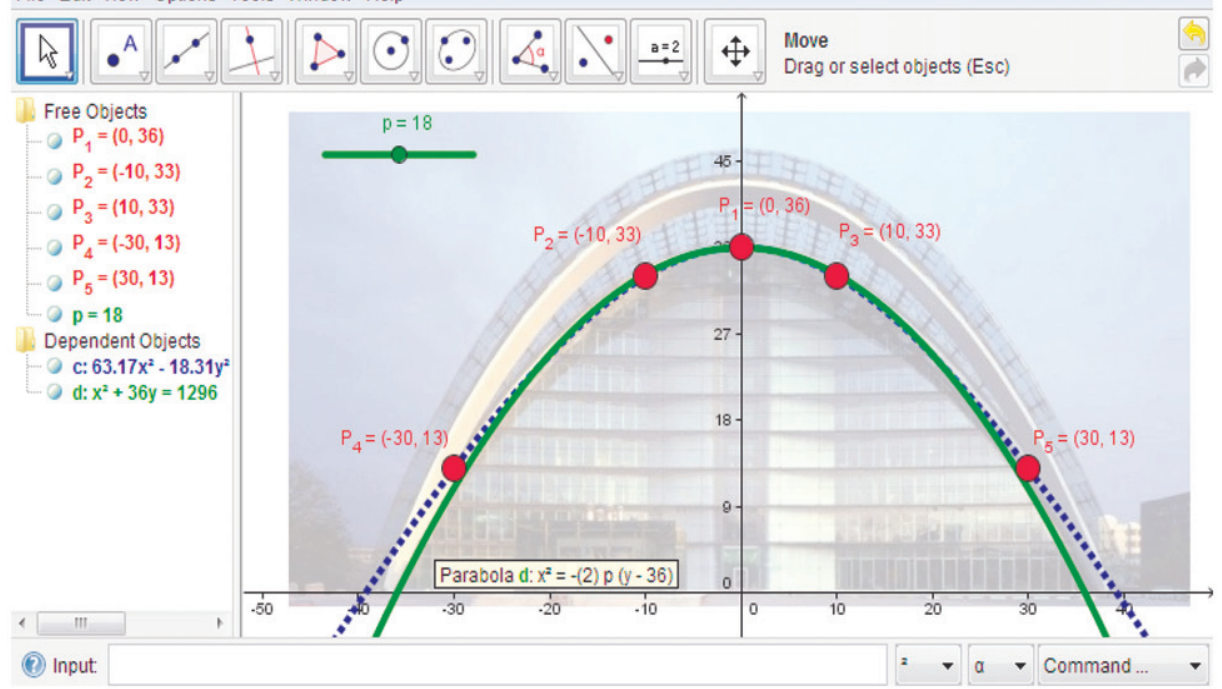

Figure 3. Approximation of the parabolic arc using a slider related with the focal distance.

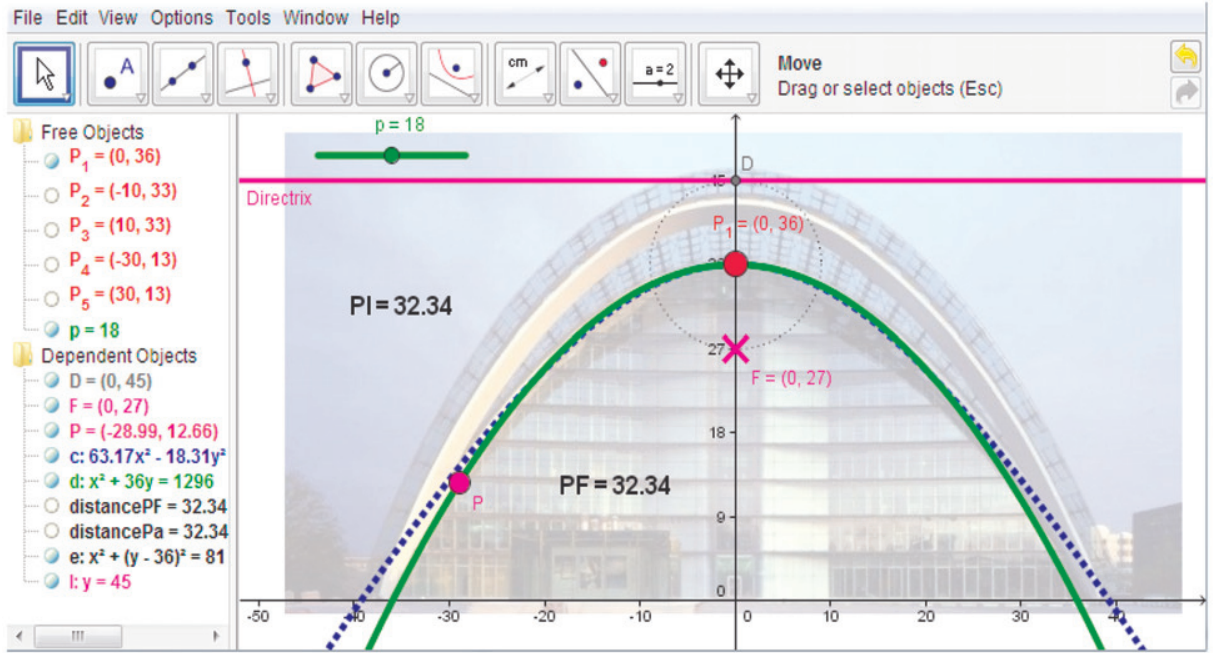

Figure 4. Directrix and focus of the parabolic arc.

question was asked by one of our students: Would it be possible to obtain the focus and the directrix line of our parabola without knowing neither its equation nor its focal distance? It would seem that the answer should be no, but surprisingly, after a spontaneous debate, the use of dynamic geometry gave us a positive answer. The procedure in our example would be the following (Figure 5).

(i) Since the focus of our parabola is in its axis $x=0$, let us create a slider $f$ in the interval $[0,36]$ and let us define the point $A=(0, f)$, which will be our candidate to focus. 


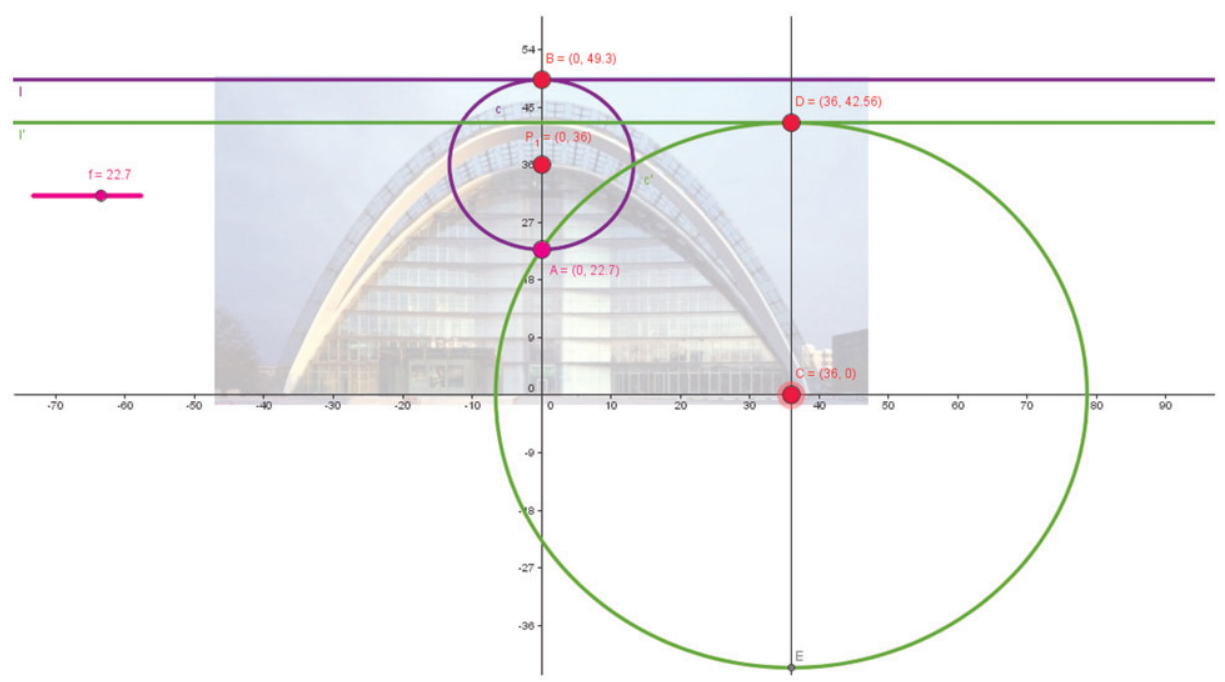

Figure 5. Obtention of focus and directrix using dynamic geometry.

(ii) Let us define the circumference $c$ of centre $(0,36)$ passing through $A$ and let $B$ be the second intersection point of $c$ with the axis $x=0$. The straight line $l$ which passes through $B$ and is parallel to the coordinate axis is our candidate to directrix line.

(iii) Let us define a second point of the arc, for instance, $C=(36,0)$. Since its distance to the focus has to be the same as its distance to the directrix line, we define the circumference $c^{\prime}$ of centre $C$ which passes through $A$. Let us define the straight line $x=36$ and let $D$ be the intersection point between $c^{\prime}$ and this last line. The straight line $l^{\prime}$ which passes through $D$ and is parallel to the coordinate axis would also be the directrix line.

(iv) Finally, it is enough to move the slider $f$ in order to obtain both lines $l$ and $l^{\prime}$ to be the same. It can be checked that it happens if and only if $f=27$.

\section{3D modeling in a 2D-DGS}

Let $O$ be the origin of coordinates of two orthogonal reference systems $O X Y Z$ and $O X^{\prime} Y^{\prime} Z^{\prime}$ and let $l$ be the straight line which is the intersection between the planes $O X Y$ and $O X^{\prime} Y^{\prime}$, when they are different. The orientation between both systems is uniquely determined by three angles: the angle $\alpha$, formed by the straight lines $O Z$ and $O Z^{\prime}$, the angle $\beta$, formed by the straight lines $O X^{\prime}$ and $l$, and the angle $\gamma$, formed by the straight lines $l$ and $O X$. If $O X Y$ coincides with $O X^{\prime} Y^{\prime}$, then $\beta=\gamma$ is the angle formed by $O X$ and $O X^{\prime}$. The variation of these three angles implies the movement of the second system of reference with respect to the first one and any rigid object whose coordinates are given with respect to the mobile system will be moved in the same way. Specifically, $\alpha$ determines the inclination of the rigid object with respect to the fixed system $O X Y Z$ and $\beta$ determines the rotation in the plane $O X^{\prime} Y^{\prime}$. As a consequence, any three-dimensional rigid object can be displayed by any 


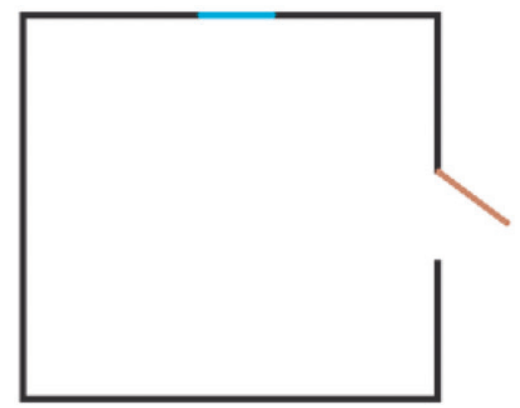

Figure 6. Floor plan to be elevated.

orthographic projection which depends on the Euler angles and whose focus is fixed at infinite distance.

Even if GeoGebra does not work yet with three-dimensional tools, it is possible to use its algebraic nature in order to define an orthographic projection based on the Euler's angles. Keeping in mind the work ${ }^{9}$ of Professor G. Tulloue in Cabri, Arranz et al. [9] define in GeoGebra the following orthogonal projection of a point $P=(a, b, c)$ :

$$
\pi_{r}(P)=(r \cdot(a \cdot \sin (\beta)+b \cdot \cos (\beta)), r \cdot(-a \sin (\alpha) \cos (\beta)+b \cdot \sin (\alpha) \sin (\beta)+c \cdot \cos (\alpha))),
$$

where $r$ determines the scale of the projection. If $P$ is defined as the list $P=\{a, b, c\}$, the projection $\pi_{r}$ can be implemented in GeoGebra in such a way that it can depend on three (previously defined) sliders $r, \alpha$ and $\beta$. It is enough to define in the input box the related point:

$$
\begin{aligned}
P^{\prime}= & (r(\text { Element }[P, 1] \sin (\beta)+\text { Element }[P, 2] \cos (\beta)), r(- \text { Element }[P, 1] \sin (\alpha) \cos (\beta) \\
& + \text { Element }[P, 2] \sin (\alpha) \sin (\beta)+\text { Element }[P, 3] \cos (\beta))) .
\end{aligned}
$$

A new tool $O S P$ (Orthogonal Scaled Projection), with input parameters $\{a, b, c\}$, $r, \alpha$ and $\beta$, is then defined as a macro so that a $3 \mathrm{D}$ display of points can be possible. We want our students to define it instead of giving it to them, because with this simple construction, they start to understand the underlying mathematical tools on which CAD systems are based. An easy application is the elevation of a floor plan (Figure 6) using the new command OSP and the tool Polygon:

(i) Given two points $P_{1}$ and $P_{2}$ in the space and two heights $h_{1}$ and $h_{2}$, we can project $\underset{P}{\longrightarrow}$ vertical $\underset{\left(0,0, h_{1}\right)}{\longrightarrow}$ as $P_{2}+$ a polygon of vertices $P_{1}+\overrightarrow{\left(0,0, h_{1}\right)}, P_{2}+\overrightarrow{\left(0,0, h_{1}\right)}, P_{2}+\overrightarrow{\left(0,0, h_{2}\right)}$ and $P_{1}+\overrightarrow{\left(0,0, h_{2}\right)}$. This construction can be implemented as a macro in a tool called Elevation which has as input the parameters $r, \alpha, \beta, h_{1}, h_{2}, P_{1}$ and $P_{2}$.

(ii) Once the floor plan is included in the worksheet as a background picture, it is possible to mark the vertices of all the walls so that the command Elevation can be used on them (Figure 7). A study of the design can then be easily done by moving the set of vertices on the floor plan.

\section{Differential geometry on space curves}

The point-by-point projection of Tulloue can be extended in a natural way in order to project space curves (Figure 8). In this sense, students can define a new tool 
File Edit View Options Tools Window Help

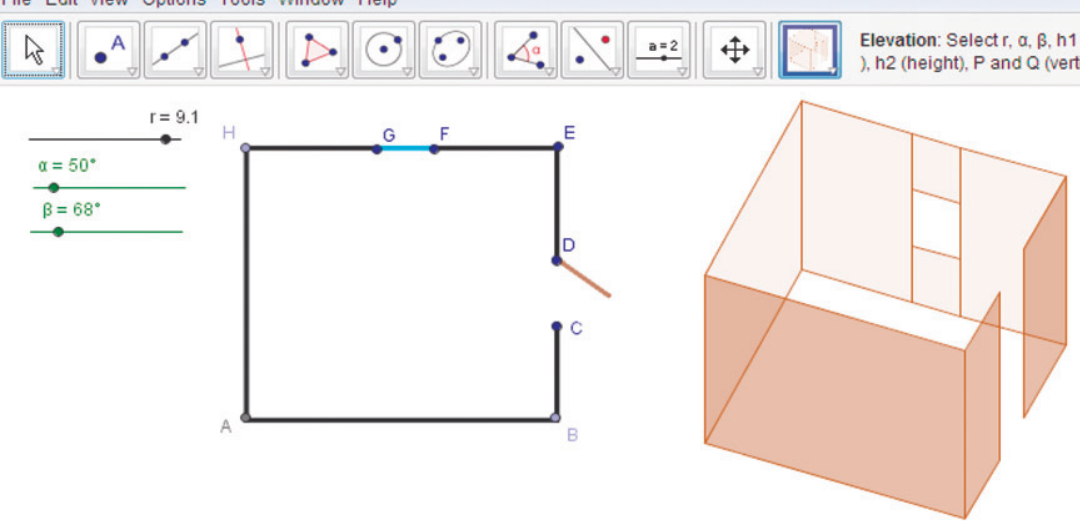

Figure 7. Floor plan elevation.

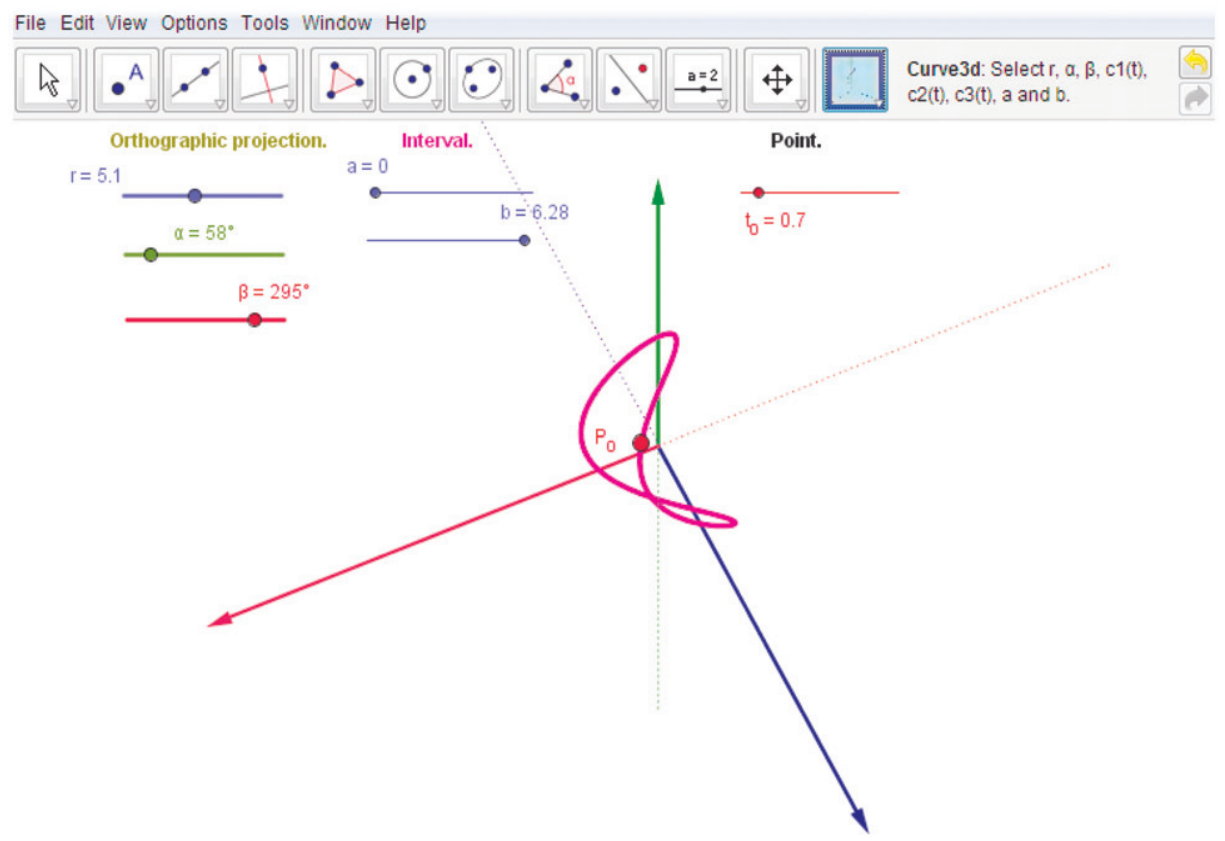

Figure 8. Space curve.

Curve $3 D$ which would be a macro such that, given a curve $C(t)=\left(c_{1}(t), c_{2}(t), c_{3}(t)\right)$ defined in an interval $[a, b]$, it executes the command Curve $[r \cdot(c 1(t) \sin (\beta)+$ $c 2(t) \cos (\beta)), r \cdot(-c 1(t) \sin (\alpha) \cos (\beta)+c 2(t) \sin (\alpha) \sin (\beta)+c 3(t) \cos (\alpha)), t, a, b]$. Once the curve is projected, points can be created on it and, since GeoGebra calculates derivative functions, local properties of $C(t)$ can be then studied. However, some previous commands have to be defined like those corresponding to the norm (Norm) and the normalization (Normalize) of a vector or the inner product (InnerProduct) 


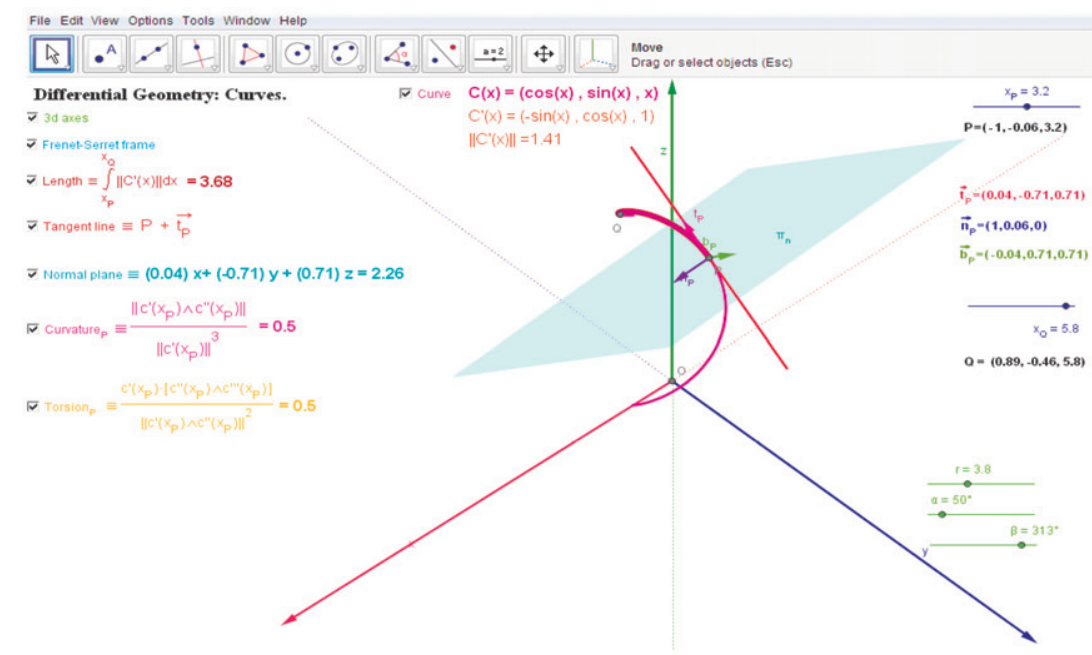

Figure 9. Differential Geometry on curves in GeoGebra.

and the cross product (CrossProduct) of two vectors. Thus, if a slider $t_{0}$ is defined in $[a, b]$ and the point $P=C\left(t_{0}\right)$ is projected, then concepts related to differential geometry on space curves can be dynamically implemented (Figure 9). Let us see several examples which our students have to define as macros. Previously, they define the lists $D C_{P}=\left\{c 1^{\prime}\left(t_{0}\right), c 2^{\prime}\left(t_{0}\right), c 3^{\prime}\left(t_{0}\right)\right\}, D 2 C_{P}=\left\{c 1^{\prime \prime}\left(t_{0}\right), c 2^{\prime \prime}\left(t_{0}\right), c 3^{\prime \prime}\left(t_{0}\right)\right\}$ and $D 3 C_{P}=\left\{c 1^{\prime \prime \prime}\left(t_{0}\right), c 2^{\prime \prime \prime}\left(t_{0}\right), c 3^{\prime \prime \prime}\left(t_{0}\right)\right\}$.

(i) The Frenet frame of $C$ at $P$ can be obtained by defining the tangent, the normal and the binormal vectors:

$$
\begin{gathered}
t_{P}=\operatorname{Vector}\left[P, O S P\left[P+\operatorname{Normalize}\left[D C_{P}\right], r, \alpha, \beta\right]\right], \\
n_{P}=\operatorname{Vector}\left[P, O S P\left[P+\operatorname{Normalize}\left[D 2 C_{P}\right], r, \alpha, \beta\right]\right], \\
b_{P}=\operatorname{Vector}\left[P, O S P\left[P+\operatorname{Normalize}\left[\operatorname{Cross} P \operatorname{Oroduct}\left[t_{P}, n_{P}\right]\right], r, \alpha, \beta\right]\right] .
\end{gathered}
$$

(ii) The tangent line of $C$ at $P$ can be projected as

Curve $3 D\left[r, \alpha, \beta, c_{1}\left(t_{0}\right)+c_{1}^{\prime}\left(t_{0}\right) x, c_{2}\left(t_{0}\right)+c_{2}^{\prime}\left(t_{0}\right) x, c_{3}\left(t_{0}\right)+c_{3}^{\prime}\left(t_{0}\right) x,-2,2\right]$.

(iii) The normal plane of $C$ at $P_{0}$ can be projected as a polygon with vertices

$$
\begin{array}{ll}
A=O S P\left[P+4 n_{P}+4 b_{P}, r, \alpha, \beta\right], & B=O S P\left[P+4 n_{P}-4 b_{P}, r, \alpha, \beta\right], \\
C=O S P\left[P-4 n_{P}-4 b_{P}, r, \alpha, \beta\right], & D=O S P\left[P-4 n_{P}+4 b_{P}, r, \alpha, \beta\right] .
\end{array}
$$

(iv) The curvature and the torsion of $C$ at $P$ are obtained as

$$
\begin{gathered}
\kappa_{P}=\operatorname{Norm}\left[\text { CrossProduct }\left[D C_{P}, D 2 C_{P}\right]\right] / \operatorname{Norm}\left[D C_{P}\right]^{3} . \\
\tau_{P}=\operatorname{InnerProduct}\left[D C_{P}, \operatorname{CrossProduct}\left[D 2 C_{P}, D 3 C_{P}\right]\right] / \\
\operatorname{Norm}\left[\operatorname{CrossProduct}\left[D C_{P}, D 2 C_{P}\right]\right]^{2} .
\end{gathered}
$$

(v) Given a second point $Q=C\left(t_{1}\right)$, it is possible to define the length of the curve $C$ between the points $P$ and $Q$ :

$$
\text { Integral }\left[\operatorname{sqrt}\left(c 1^{\prime}(x)^{2}+c 2^{\prime}(x)^{2}+c 3^{\prime}(x)^{2}\right), t_{0}, t_{1}\right] \text {. }
$$




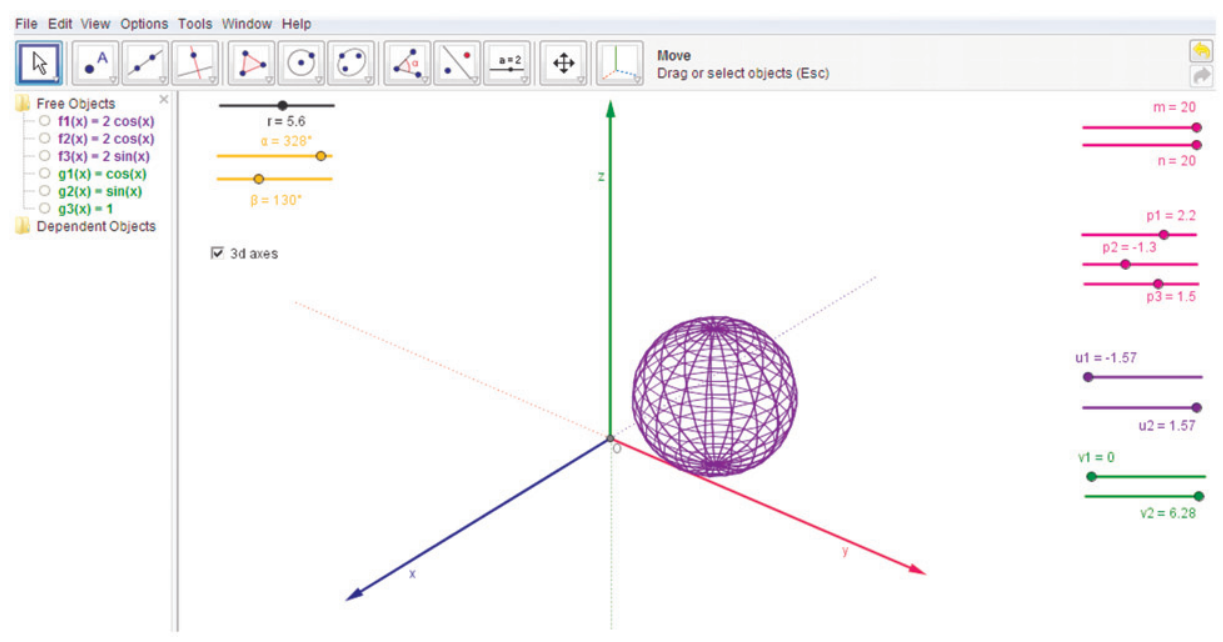

Figure 10. Surface in GeoGebra.

Unlike CAD systems, once the previous tools are defined, students can dynamically obtain, by moving only the slider $t_{0}$, local information of a given curve, like, for instance, when the tangent line has a given direction or which are the points of maximum and minimum curvature or torsion. Several properties about space curves can also be dynamically checked like the fact that circular helices have constant curvature and torsion. Some tasks based on realistic problems are also asked: "A curved wall of null torsion is going to be built by following the curve $\alpha(t)=\left(2 t, 4(t-1)^{2}, 2 t\right)$. In order to have a solid wall, it is necessary to build a concrete pillar under the point of maximum curvature. Check that the torsion of $\alpha$ is zero and that the corresponding binormal vector is constant. Indicate also the exact position of the pillar."

\section{Modeling surfaces}

Analogously to CAD systems, surfaces can be defined in GeoGebra as a mesh of polylines (Figure 10). However, unlike CADs, its algebraic tools makes possible the use of parametric equations. In our courses, given a point $P=\left(p_{1}, p_{2}, p_{3}\right)$ and two functions $f(x)=\left(f_{1}(x), f_{2}(x), f_{3}(x)\right)$ and $g(x)=\left(g_{1}(x), g_{2}(x), g_{3}(x)\right)$, we are interested in the following two types of surfaces:

(i) Those surfaces defined as

$$
S(u, v)=P+f(u) \cdot g(v) .
$$

(ii) Ruled surfaces

$$
S(u, v)=f(u)+v \cdot g(u) .
$$

Cylinders, spheres, paraboloids, cones, hyperboloids, tori and astroids are surfaces of the first type. Conoids and cylindrical and conical surfaces can be 


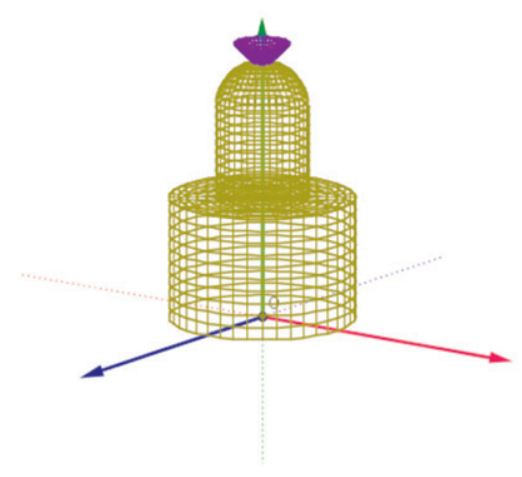

Figure 11. $3 D$ model of a tower with a dome.

obtained as ruled surfaces. Thus, the implementation of a general tool which projects these two kinds of surfaces by starting from their parametric equations would make possible to model almost any type of architectural structure. To define it, let us suppose that both kind of surfaces are defined in $\left[u_{1}, u_{2}\right] \times\left[v_{1}, v_{2}\right]$. The mesh of curves will then be given by a set of $m u$-parametric curves and $n v$-parametric curves. First, we have to define the sets of intersection nodes, which will be, respectively, obtained as

$$
\begin{gathered}
\text { Sequence[Sequence }\left[\operatorname{OSP}\left[\left\{p_{1}+f_{1}(u) g_{1}(v), p_{2}+f_{2}(u) g_{2}(v), p_{3}+f_{3}(u) g_{3}(v)\right\}, r, \alpha, \beta\right],\right. \\
\left.\left.v, v_{1}, v_{2},\left(v_{2}-v_{1}\right) /(n-1)\right], u, u_{1}, u_{2},\left(u_{2}-u_{1}\right) /(m-1)\right] .
\end{gathered}
$$

$$
\begin{gathered}
\text { Sequence[Sequence }\left[\operatorname{OSP}\left[\left\{f_{1}(u)+v g_{1}(u), f_{2}(u)+v g_{2}(u), f_{3}(u)+v g_{3}(u)\right\}, r, \alpha, \beta\right],\right. \\
\left.\left.v, v_{1}, v_{2},\left(v_{2}-v_{1}\right) /(n-1)\right], u, u_{1}, u_{2},\left(u_{2}-u_{1}\right) /(m-1)\right] .
\end{gathered}
$$

If we denote by $N$ the corresponding list, the $u$-and $v$-parametric curves are then drawn by writing

$$
\begin{aligned}
C_{u}= & \text { Sequence[Sequence[Segment[Element }[\text { Element }[N, i], j], \\
& \text { Element[Element }[N, i+1], j]], i, 1, m], j, 1, n+1] . \\
C_{v}= & \text { Sequence[Sequence[Segment[Element[Element }[N, i], j], \\
& \text { Element[Element }[N, i], j+1]], i, 1, m], j, 1, n] .
\end{aligned}
$$

Once both macros are defined, it is easy to create in the same worksheet a set of surfaces which can model an architectural structure. Indeed, it is possible to define specific tools corresponding to common elements like pillars (cylinders) or domes (semi spheres). As a first task, students are asked to build a cylindrical tower of two floors, with given dimensions, such that a dome covers its top and there exists a sculpture on the dome which seems a truncated cone (Figure 11). Unlike CAD systems, students have to find the exact parametric equations of all the related surfaces in order to model the tower. The use of sliders related to some of the measurements, like radius or height, can be useful in order to obtain them in a dynamical way. Moreover, sliders related to the number of $u$ - and $v$-parametric curves makes it possible to model some constructions whose parametric equations 


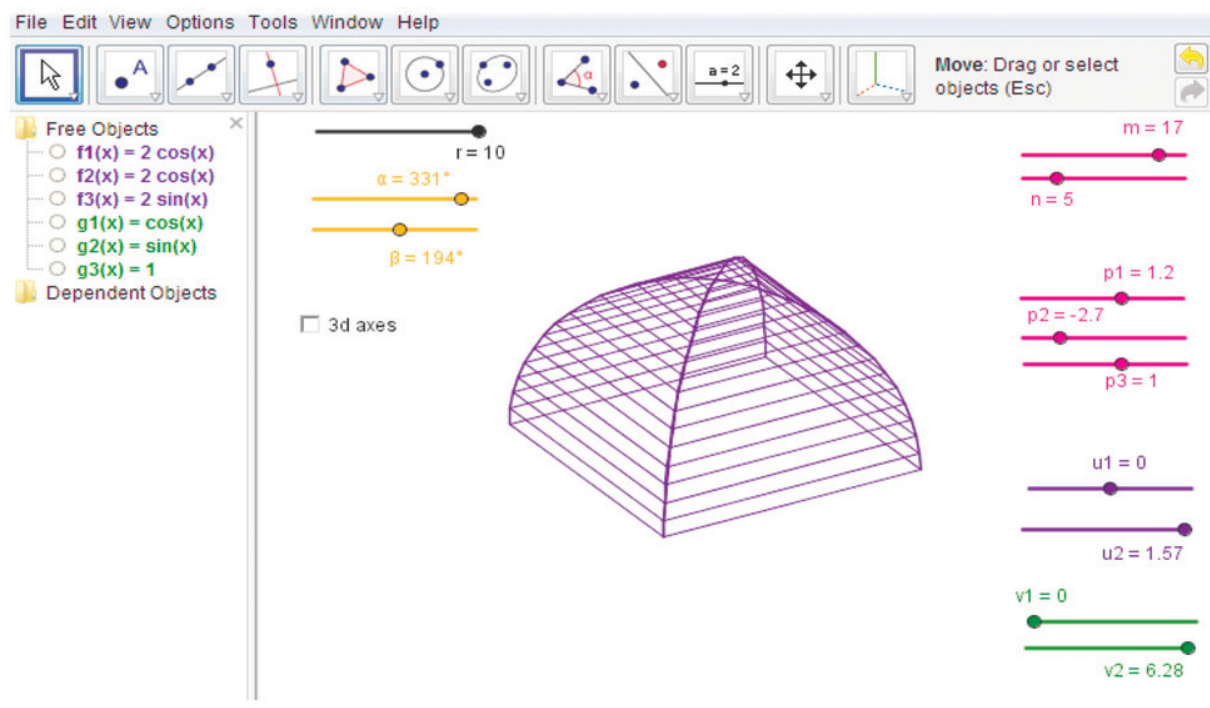

Figure 12. $3 D$ model of a cloister vault.

(a)

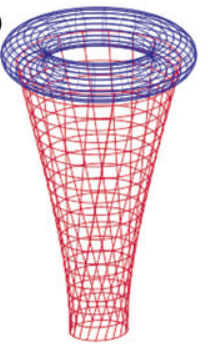

(d)

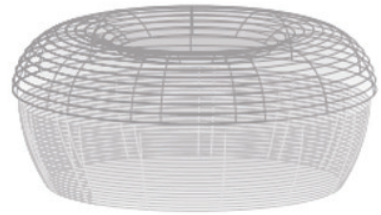

(b)

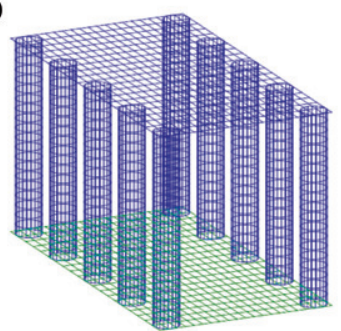

(e) (c)

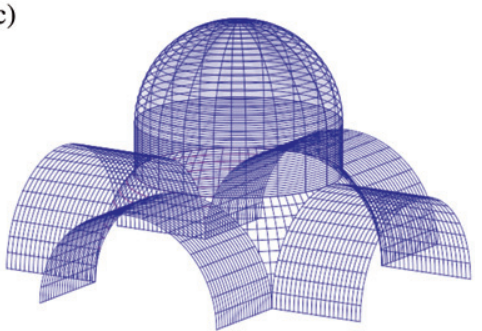

(f)
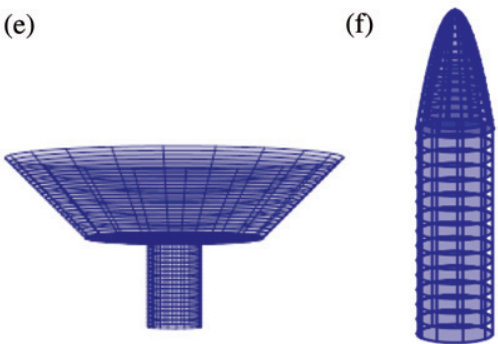

Figure 13. 3D models of real architectural structures: (a) Water tower in Ciechanów, Poland; (b) Parthenon in Athens, Greece; (c) Dome of Saint Peter in Vatican City; (d) Legal Medicine Institute, Campus of Justice in Madrid, Spain; (e) Niterói Contemporaneum Art Museum, Brazil; and (f) 30 St. Mary Axe in London, England.

would need a dependence between parameters. This is the case, for instance, of the cloister vault (Figure 12), which can be obtained by projecting a semi sphere with only $5 u$-parametric curves.

As a final task, students have to find and model some real architectural structures which consist of at least two surfaces. In Figure 13 we show some of their works. 


\section{Final remarks}

We have seen in this article that, although the final result is not so visually impressive, macros and sliders can be used to turn GeoGebra into a dynamic parametric CAD system. Students of Architecture and Building Engineering Degrees can work in a familiar environment and observe some of the mathematical concepts and tools on which are based CAD systems. The implementation and experience in two mathematical courses during the Academic Year 2009-10 has shown that a methodology based on a CAS/DGS facilitates to capture the attention of students and improve their attitudes towards Mathematics.

\section{Notes}

1. http://www.autodesk.com/autocad

2. http://www.graphisoft.com/products/archicad/

3. http://www.rhino3d.com/

4. http://www.cabri.com

5. http://www.cinderella.de

6. http://www.geogebra.org

7. http://www.maplesoft.com

8. All the used commands have been tested again in the last official version GeoGebra 3.2.45.0.

9. http://gtulloue.free.fr/Cabri3D/euler/euler.html

\section{References}

[1] H.K. Banerjee and E. de Graaff, Problem-based learning in architecture: Problems of integration of technical disciplines, Eur. J. Eng. Edu. 21(2) (1996), pp. 185-195.

[2] E. de Graaff and P.A.J. Bouhuijs (eds.), Implementation of Problem-Based Learning in Higher Education, Thesis publishers, Amsterdam, 1993. ISBN: 9051702094.

[3] I. Verner and S. Maor, The effect of integrating design problems on learning mathematics in an architecture college, Nexus Netw. J. 5(2) (2003), pp. 111-115.

[4] I. Verner and S. Maor, Mathematical mode of thought in architecture design education: A case study, Nexus Netw. J. 8(1) (2006), pp. 93-106.

[5] H. Freudenthal, Mathematics as an Educational Task, D. Reidel, Dordrecht, 1973.

[6] A. Howson, J. Kahane, P. Laugine, and E. Turckheim (eds.), Mathematics as a Service Subject, Cambridge University Press, Cambridge, 1988, ICMI Study Series.

[7] I. Verner and S. Maor, Mathematical aspects in an arquitectural design course: The concept, design assignments, and follow-up, Nexus Netw. J. 9(2) (2007), pp. 363-375.

[8] I. Verner and S. Maor, Integrating design problems in mathematics curriculum: An architecture college case study, Int. J. Math. Educ. Sci. Technol. 32(6) (2001), pp. 817-828.

[9] J.M. Arranz, R. Losada, J.A. Mora, T. Recio, and M. Sada, Geogebra on the rocks, in Model-centered Learning with GeoGebra: Theory and Practice in Mathematics Education, L. Bu and R.C. Schoen, eds., Sense Publishers, Rotterdam, The Netherlands, 2010, to appear. 